\title{
Acoustic emissions in compression of building materials: $q$-statistics enables the anticipation of the breakdown point
}

\author{
A. Greco ${ }^{1}$, C. Tsallis ${ }^{2,3,4}$, A. Rapisarda ${ }^{4,5,6}$, A. Pluchino ${ }^{5,6}$, G. Fichera ${ }^{1}$, L. Contrafatto ${ }^{1}$ \\ 1 Department of Civil Engineering and Architecture - University of Catania, Italy \\ ${ }^{2}$ CBPF, Rio de Janeiro, Brazil \\ 3 Santa Fe Institute, NM, USA \\ 4 Complexity Science Hub Vienna, Austria \\ 5 Department of Physics and Astronomy "Ettore Majorana" - University of Catania, Italy \\ ${ }^{6}$ INFN Section of Catania, Italy
}

(Dated: January 9, 2019)

\begin{abstract}
In this paper we present experimental results concerning Acoustic Emission (AE) recorded during cyclic compression tests on two different kinds of brittle building materials, namely concrete and basalt. The AE inter-event times were investigated through a non-extensive statistical mechanics analysis which shows that their decumulative probability distributions follow q-exponential laws. The entropic index $q$ and the relaxation parameter $\beta_{q} \equiv 1 / T_{q}$, obtained by fitting the experimental data, exhibit systematic changes during the various stages of the failure process, namely $\left(q, T_{q}\right)$ linearly align. The $T_{q}=0$ point corresponds to the macroscopic breakdown of the material. The slope, including its sign, of the linear alignment appears to depend on the chemical and mechanical properties of the sample. These results provide an insight on the warning signs of the incipient failure of building materials and could therefore be used in monitoring the health of existing structures such as buildings and bridges.
\end{abstract}

Keywords: Acoustic emissions, fracture process, failure prediction, q-statistics.

The study of Acoustic Emission (AE) due to applied loads is a powerful technique for deeply understanding the dynamics of the fracture processes and the behavior of damage propagation in different kind of materials approaching failure [1-4]. The investigation in controlled laboratory tests allows obtaining valuable information which could be very important in a health monitoring strategy of large structures [5, 6]. Furthermore, several studies introduce AE as a useful non destructive technique also capable of detecting internal defects on structural members [7]. The phenomenon of AE has also many interesting similarities with the investigation of seismic signals in earthquakes dynamics. Although the scales are different, both the phenomena are connected to the release of elastic energy from sources inside a medium, a structural material in one case and the earth in the other case. In general AE events occur when sequential series of short mechanical waves are released due to the activation of internal micro-fractures caused by applied mechanical loads. The released elastic waves travel as spherical waves within the bulk of the material. In brittle materials, such as the ones considered in the present study, AEs are produced by the growth of local micro-cracks associated with the fracture phenomenon and provide a great variety of information on the development of the failure. Coalescence and nucleation of these cracks cause the failure and produce acoustic emissions on different spatial and temporal scales. These emissions therefore represent indicators of the phenomena occurring within the material, from the breaking of atomic bonds to macroscopic structural defects. The detection of the AEs can therefore allow to know how the damage accumulates within the material and to predict its failure.
The application of the time series statistical analysis to the investigation of AEs of loaded building materials is quite recent, although in the last decades several studies have shown the potential of such a technique in other quite similar contexts, such as earthquakes dynamics, crumpled plastic sheets and financial time series [813]. In these fields, non-extensive generalized statistical mechanics has also proved to be particularly effective in describing universal features emerging at criticality or at the edge of chaos [14-20]. Along this line, in this paper we perform a statistical investigation of AEs occurring in relatively small cubic specimens of concrete and basalt subjected to compressive cyclic loadings. In particular, we analyze the decumulative probability density functions of inter-event time series of the $\mathrm{AE}$ recordings and characterize them by means of $q$-exponential curves typical of the generalized statistics. The parameters extracted from the fits are also compared to analogous ones concerning other building materials and reported in the literature [1]. The resulting curves suggest a possible universal behavior characteristic of different kinds of materials approaching failure and could therefore be used to obtain useful information on the fracture processes in existing structures.

Concrete is an artificial conglomerate consisting of a mixture of binder (cement), water and fine and coarse aggregates (sand and gravel) according to appropriate ratios. It is characterized by having an excellent compression behavior, resistance to water and atmospheric agents, while it has low tensile and flexural strength. Basalt is an effusive rock of volcanic origin, resulting from the escape of magma. The rapid reduction in pressure and the cooling caused by contact with the atmo- 
sphere stop the crystallization process, giving this rock a very compact structure with a dark microcrystalline paste characterized by a low silica content. Basalt has excellent technical characteristics not subject to alterations, high degree of compactness with excellent resistance to mechanical stresses and impacts, low gelling. For both materials, cubic specimens of $150 \mathrm{~mm}$ side were prepared in a Laboratory of the Department of Civil Engineering and Architecture of the University of Catania. The basalt came from the quarries on the Etna volcano while, the concrete specimens have been prepared in the laboratory with the following percentage of components: $25 \%$ sand, $41 \%$ gravel, $13 \%$ water, $21 \%$ cement. For the preparation of the concrete specimens a commercial Portland cement, type CEM II/B-LL 32,5R, according to European Norm EN-197-1, was used. The tests on the concrete samples were carried out after the necessary 28 days from the packaging date in order to allow the hardening of the material.

Acoustic emissions during the tests were measured through a "Piezotron Acoustic Emission Sensor" mounted on the specimen and connected to a "AEPiezotron Coupler", which supplies power to the sensor and processes the output signals. The sensor is made of a piezoelectric sensing element mounted on a thin steel diaphragm coupling surface, and is capable to measure emissions of surface and longitudinal waves over a broad high frequency range, $50-400 \mathrm{kHz}$. The sensing element is acoustically isolated from the sensor housing and therefore well protected against external noise. The coupler is provided with a built-in root mean square (RMS) converter whose integration time constant can be freely selected; even the bandpass filter frequency limits and the gain are both selectable. The sensor was mounted with a stud onto the surface of the specimen and a thin layer of silicone grease was put between the coupling surfaces. The analogic output RMS signal, obtained with $1.2 \mathrm{~ms}$ integration time constant, was recorded by a data acquisition front end and later post processed.

The cyclic compression tests, with load control, have been performed on several cubic specimens of both the chosen materials using a $5000 \mathrm{kN}$ hydraulic press connected to a data acquisition unit. The software allows to set all the necessary test parameters, such as the values of the load sequence, its speed of application and the peak sensitivity, that is the sudden decrease in load at the occurrence of which the test is stopped since the specimen is

\begin{tabular}{|c|c|c|}
\hline Material & Load step $(\mathrm{kN})$ & $\begin{array}{c}\text { Average } \\
\text { Failure } \\
\text { load }\end{array}$ \\
\hline Concrete & $\begin{aligned} 0->900->800 & >950 \\
& >>800->1000->800->1050 \rightarrow>800 \rightarrow 1100->800 \\
& \rightarrow 1150 \rightarrow 800 \rightarrow 1200\end{aligned}$ & 1154 \\
\hline Basalt & 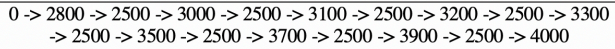 & 3829 \\
\hline
\end{tabular}

FIG. 1: Table with the loading values of the compression cyclic tests.
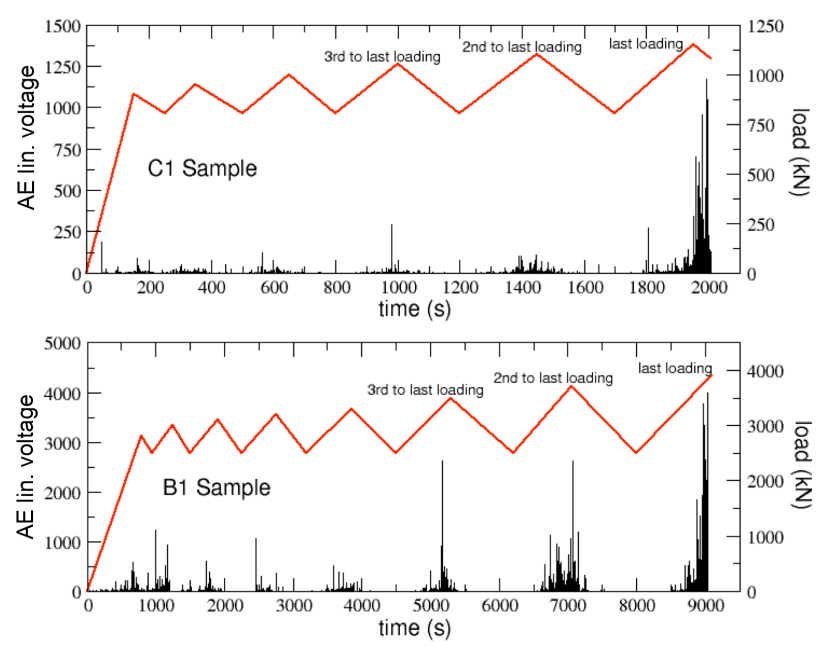

FIG. 2: Experimental recording of the AE linear voltage as a function of time during the cyclic compression test for two samples of, respectively, concrete $(\mathrm{C} 1$, top panel) and basalt (B1, bottom panel), see text.

assumed to be broken. Table in Figure 1 reports the typical values of the sequences of applied loads together with the average failure load for the two considered materials. In each loading and unloading step (except for the first loading starting from zero, which is faster), a progressive increment rate of $0.05 \mathrm{MPa} / \mathrm{s}$ has been selected.

In Figure 2 two examples of the acoustic emissions during the complete cyclic test for a couple of specimens of both concrete ( $\mathrm{C} 1$, top panel) and basalt (B1, bottom panel), are reported. The loading and unloading curves in the two cases are also plotted for comparison. It is worth noticing that, in the case of the concrete specimens, the acoustic emissions started from high values of the applied load and were concentrated at the load peaks. On the other hand, in basalt specimens, high intensity emissions started immediately after the application of the first loading and lasted throughout the tests. This rock, in fact, being much stiffer than concrete, produces acoustic emissions with high frequencies and therefore more easily measured by the sensor used.

Observing the graphs, it is also evident that, during each load cycle, very few emissions are recorded until the load value corresponding to the previous peak is exceeded. This is a confirmation of the well known Kaiser effect, following from the fact that the microcracks opened in the material during the loading phases do not propagate until a load intensity greater than the one previously experienced is reached. Finally, the failures for the considered samples do occur after 2007 seconds at a load value of $1080 \mathrm{kN}$ for the concrete sample $\mathrm{C} 1$ and after 9101 second at a load value of $3902 k N$ for the basalt sample B1. Figure 3 shows a concrete specimen at the end of the compression test and highlights the presence of several cracks which caused its failure.

Let us investigate, now, the inter-event time series of 


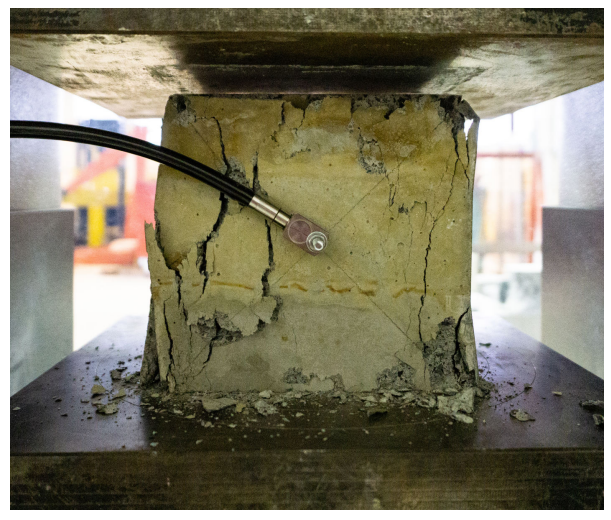

FIG. 3: A concrete specimen inside the hydraulic press after the compression test and the final rupture.

the $\mathrm{AE}$ recordings during the compression tests on some of the considered specimens. In particular, we perform our analysis on two concrete specimens, namely $\mathrm{C} 1$ and $\mathrm{C} 2$, and on two basalt specimens, B1 and B2 (notice that $\mathrm{C} 1$ and $\mathrm{B} 1$ are the same samples addressed in Figure 2), and we consider only the last three loadings, being the latter those with the best statistics.

The inter-event time $\delta \tau(t)$ is defined as the time interval between two consecutive recordings $\operatorname{AE}(n)$ and $\operatorname{AE}(n-$ 1). In other words:

$$
\delta \tau(t)=t_{A E(n)}-t_{A E(n-1)}
$$

where $t_{A E(n)}$ is the time at which the $n$-th AE event does occur and $t_{A E(n-1)}$ the time of the previous event.

In Figure 4 we show, as an example, two groups of three graphs each, concerning the compression test of the two specimens $\mathrm{C} 1$ and B1. In each group we report, from top to bottom, the AE recordings of the last loading, the corresponding inter-event time series $\delta \tau(t)$ and the probability distribution function $\mathrm{P}(\delta \tau)$. It appears that, for both the considered samples, the $\mathrm{P}(\delta \tau)$ distribution can be well fitted (in log-log scale) by power-law curves, with slope -1.6 for $\mathrm{C} 1$ and -1.9 for B1. This is a first signature of the complex behavior of the failure process, which in the following will be analyzed in terms of decumulative distribution functions.

The decumulative distribution $P(>\delta \tau)$ of the interevent time series reports, for each value of $\delta \tau$ in the interval $[0,500]$, the fraction of inter-event times greater than that value. Thus, we expect curves that (in log$\log$ scale) start from 1 for small values of $\delta \tau$ and, after a plateau, begin to go down with a certain power-law decay for higher values of $\delta \tau$. In the four panels of Figure 5 we plot, from top to bottom, the decumulative distribution functions relating to the last three loadings of the specimens C1, C2, B1 and B2. As expected, all the reported data show a power-law tail after an initial saturation. This behavior can be very well reproduced by means of a decreasing $q$-exponential function $e_{q}(\delta \tau)$ defined as

$$
e_{q}(\delta \tau)=\left[1-(1-q) \beta_{q} \delta \tau\right]^{1 /(1-q)} .
$$
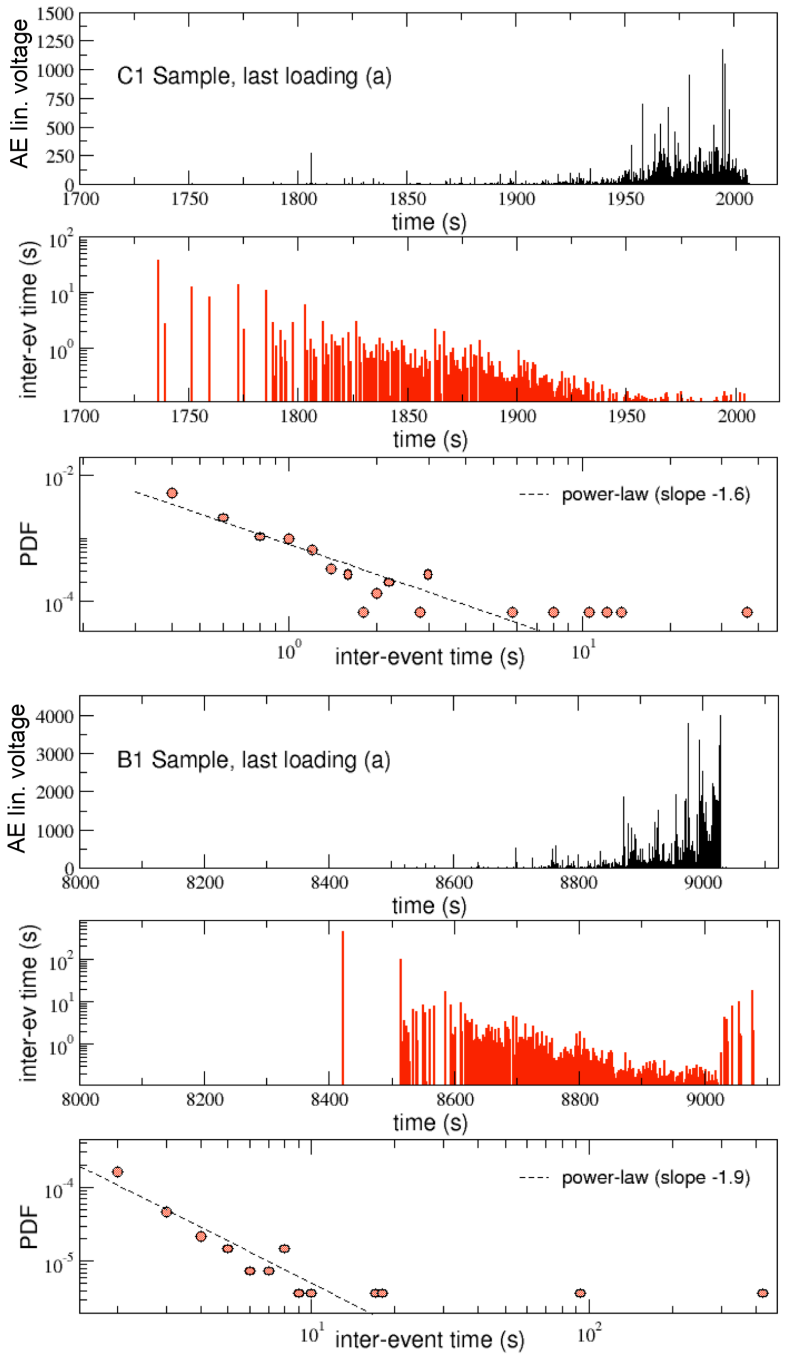

FIG. 4: The AE recordings of the last loading of the two compression test shown in Figure 2 for the two specimens C1 and $\mathrm{B} 1$ are reported, together with the inter-event time series and the corresponding PDFs.

In Eq. (2) $q$ and $\beta_{q}$ are two parameters which characterize the dynamical behavior of the system and are fixed by the fitting procedure; notice that, in the $q \rightarrow 1$ limit, we obtain $e_{1}(\delta \tau)=e^{-\beta_{1} \delta \tau}$. In the generalized statistical mechanics [16] the $q$-exponential is the function which maximizes the generalized entropy $S_{q}$ defined as

$$
S_{q}=k \frac{1-\sum_{i=1}^{W} p_{i}^{q}}{q-1}
$$

where $W$ represents the total number of microscopic configurations whose probabilities are $\left\{p_{i}\right\}$ and $k$ is the Boltzmann constant. For $q=1$ one gets the standard Boltzmann-Gibbs (BG) entropy, i.e. $S_{q=1}=S_{B G} \equiv$ $-k \sum_{i=1}^{W} p_{i} \ln p_{i}$. In general, the entropic index $q$ is considered as a measure of the strong correlations present in the system ( $q=1$ means no correlations) while $\beta_{q}$ is a sort of inverse temperature, therefore its reciprocal $1 / \beta_{q}$ 

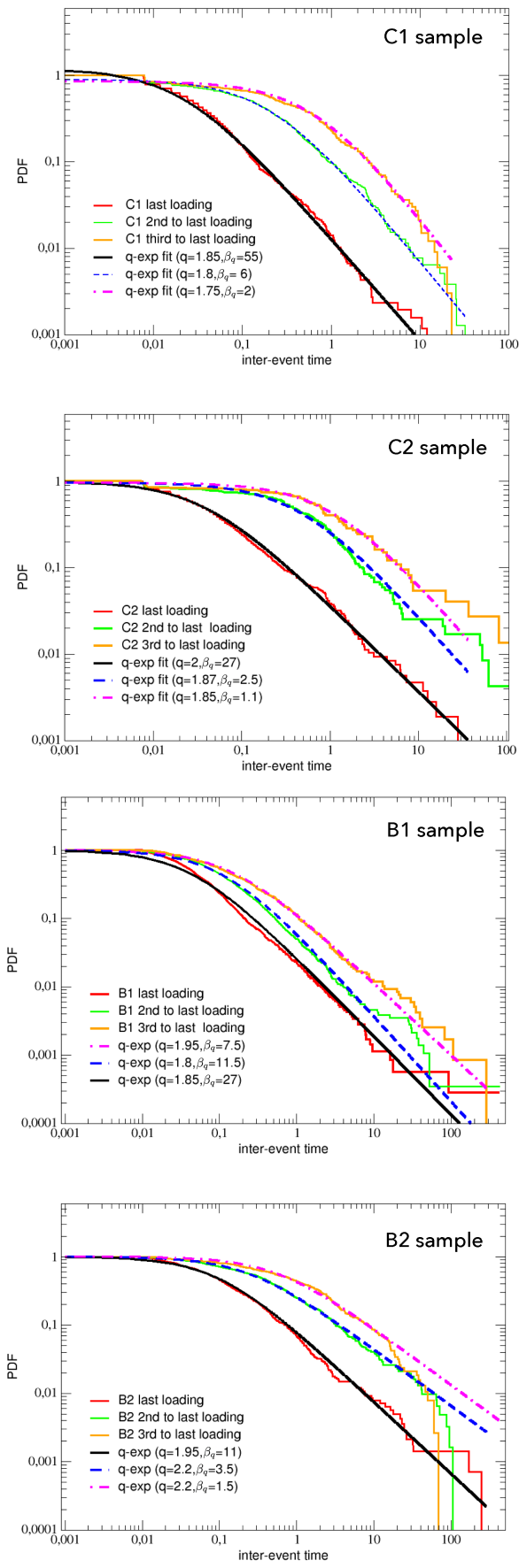

FIG. 5: From top to bottom: decumulative pdfs of the interevent times of $\mathrm{AE}$ for the last three loadings of the specimens C1, C2 (of concrete) and B1, B2 (of basalt). The fits with $q$-exponential curves are also reported, see text.

indicates the level of noise of the analyzed process. For a detailed description of the non-extensive theoretical formalism and updated information on its applications see [16].

In the context addressed in this paper, we look for the existence of a relationship between $q$ and $1 / \beta_{q}$ able to characterize the behavior of the materials in the prox-

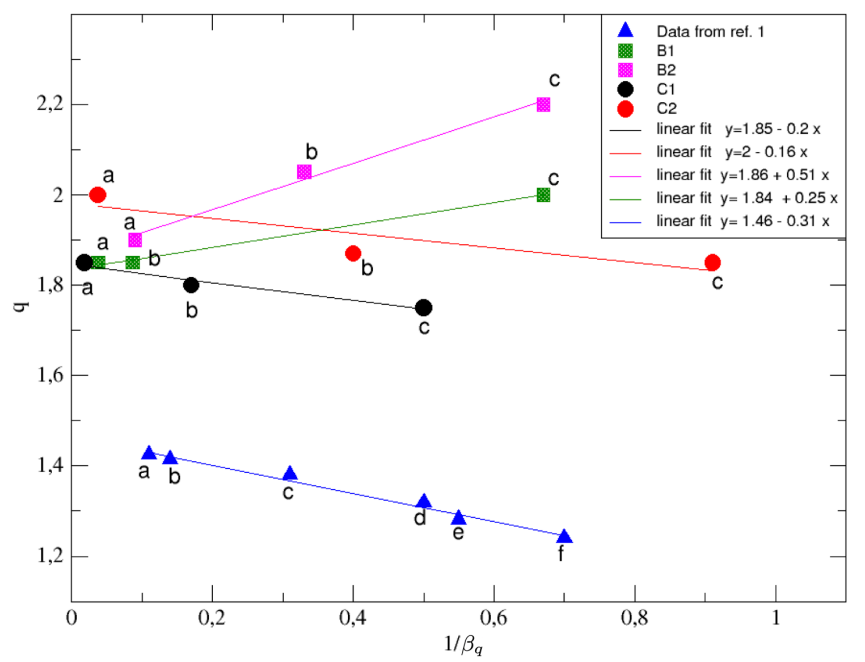

FIG. 6: The values of the entropic index of the $q$-exponential fits, reproducing the decumulative pdfs obtained from experimental data about the $\mathrm{AE}$ inter-event time series for both the concrete (C1, C2 - full circles) and basalt (B1, B2 - full squares) specimens, are reported as function of $1 / \beta_{q}$. Linear fits of the reported values are also shown. We report also (triangles) the values for $q$ and $1 / \beta_{q}$ extracted from analogous failure tests with cement mortar specimens in ref. [1], see text.

imity of the failure. Figure 6 reports a summary of the values of these parameters obtained through the $q$ exponential fits for the considered specimens C1, C2 (full circles), B1 and B2 (full squares) shown in the previous figure. Linear fits of the experimental points are also reported for helping the eye to follow their trend. The alphabetic letter below each data point refer to the order of the experimental peak loadings: $a$ for the last one, $b$ for the 2nd to last, and so on. In the same figure the results obtained in [1], with reference to $\mathrm{AE}$ recorded in white cement mortar specimens subjected to three point bending tests, have been also reported (as full triangles).

As clearly visible, for all the specimens we find that, approaching the failure, while $1 / \beta_{q}$ tends to zero, the entropic index $q$ goes towards a value in between 1.8 and 2.0. On the other hand, the trend of the linear fit is not the same for the two considered materials: in particular, approaching the failure point, it is increasing for the concrete specimens and decreasing for the basalt ones. This fact probably reflects the different behavior of the two materials while they go towards the rupture. In fact, being a composed material (see Fig.7, left panel), concrete breaks very slowly when increasing the compression, with fractures propagating along preferential lines which, as can be observed at a mesoscopic scale, surround the aggregates [21]. This phenomenon could be responsible of the long-range correlations quantified by increasing values of $q$. On the contrary, being a more compact and homogeneous natural material (see Fig.7, right panel), basalt tends to uniformly resist to stronger compressions until it suddenly breaks in a kind of explosive way: there- 


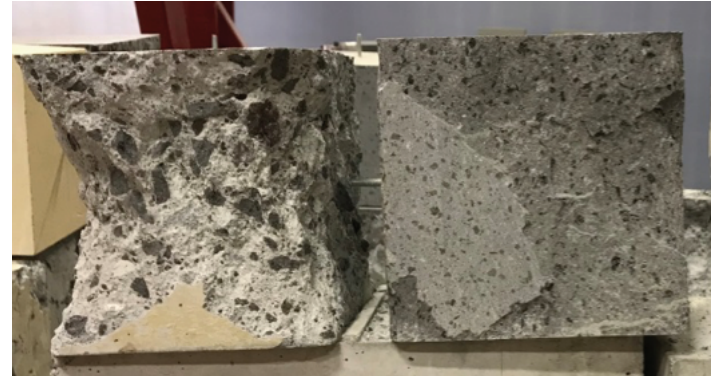

FIG. 7: Internal structure of two experimental specimen of concrete (left) and basalt (right). See text.

fore the value of $q$ decreases approaching $1 / \beta_{q}=0$.

It is interesting to compare these results with those reported in the paper of Stavrakas et al. [1]. In particular the linear fit of the $q$-exponential data for white cement mortar shows a slope similar to that obtained in the present study for concrete specimens, even if the asymptotic value of $q$ for vanishing $1 / \beta_{q}$ stays quite below the interval 1.8-2.0. This result can be due to the fact that both materials, although at different scales, are composed by a mixture of binder and aggregates which can drive the propagation of cracks along the preferential directions surrounding the aggregates. Thus, this kind of statistical analysis seems able to distinguish between at least two universality classes, parametrized by $q$ and $1 / \beta_{q}$, which include different typologies of building materials.

In conclusion, we presented the results of the $\mathrm{AE}$ recording during compression tests experiments for two kinds of building material, i.e. concrete and basalt. The obtained data have been analyzed within non-extensive statistical mechanics. In particular, in order to characterize the specific material behavior in approaching the failure point for increasing loads, we have considered the inter-event time series of AEs. The decumulative distribution functions of these AE inter-event time series show a $q$-exponential behavior with different trends of the entropic parameter $q$ as function of the relaxation parameter $\beta_{q}$ for the two kinds of considered materials. This difference is probably due to the different composition of the material which is more homogeneous in the case of basalt with respect to concrete. A comparison with previous data published in the literature, with reference to other materials, has also been discussed. The present generalized statistical analysis provides an insight on the warning signs of the incipient failure of building materials and could therefore be used in a health monitoring strategy on existing structures such as buildings and bridges.
[1] I. Stavrakas, D. Triantis, S.K. Kourkoulis, E.D. Pasiou, I. Dakanali, Latin Am. Journ. of Sol. and Struc. 13 (2016) 2283.

[2] I. Stavrakas, Frattura ed Integrità Strutturale, 40 (2017) 32-40; DOI: 10.3221/IGF-ESIS.40.03

[3] C. Stergiopoulos, I. Stavrakas, G. Hloupis, D. Triantis, F. Vallianatos, Engineering Failure Analysis, 35 (2013) 454.

[4] A. Kyriazopoulos, C. Anastasiadis, D. Triantis, F. Vallianatos, 1st Int. Conf. of the Greek Society of Exp. Mechanics of Materials, Athens, Greece, May 10-12, 2018, p.77.

[5] A. Nair, C.S. Cai, Engineering Structures 32 (2010) 1704.

[6] B. Goszczynska et al., IEEE (2012) Prognostic \& systems health management conference PHM-2012 Beijing

[7] A. Carpinteri, G. Lacidogna, F. Accornero, A.C Mpalaskas, T.E. Matikas, D.G. Aggelis Cement \& Concrete Composites 44 (2013) 9.

[8] M. S. Mega, P. Allegrini, P. Grigolini, V. Latora, L. Palatella, A. Rapisarda, and S. Vinciguerra Phys. Rev. Lett. 90 (2003) 188501.

[9] S. Abe, N. Suzuki, Eur. Phys. Lett., 65 (2004) 581.

[10] S. Lennartz, V.N. Livina, A. Bunde, S. Havlin, 81 Eur. Phys. Lett. (2008) 69001.
[11] R.S. Mendes, L.C. Malacarne, R.P.B. Santos, H.V Ribeiro, S. Picoli jr., Eur. Phys. Lett., 92 (2010) 29001.

[12] F. Lillo, R.N. Mantegna, Phys. Rev. E, 68 (2003) 016119.

[13] M.I. Bogachev, A. Bunde, Phys. Rev. E 78 (2008) 036114.

[14] F. Caruso, A. Pluchino, V. Latora, S. Vinciguerra, A. Rapisarda, Phys. Rev. E 75 (2007) 055101 (R).

[15] A. Celikoglu, U. Tirnakli, Acta Geophysica 60 (2012) 3.

[16] C. Tsallis, Introduction to nonextensive statistical mechanics - Approaching a complex world, Berlin, (2009) Springer.

[17] A. Pluchino, A. Rapisarda, C.Tsallis, Phys. Rev. E 87 (2013) 022910.

[18] G. Combe, V. Richefeu, M. Stasiak, A.P.F. Atman, Phys. Rev. Lett. 115 (2015) 238301.

[19] S.G.A. Brito, L.R. da Silva, C. Tsallis, Scientific Reports 6 (2016) 27992 .

[20] U. Tirnakli, E.P. Borges, Scientific Reports 6 (2016) 23644.

[21] A.M. Tarantino, J. Kaplunov, R. Luciano, C. Majorana, T.C. Rousakis, K. Willam, Modelling and Simulation in Engineering (2017) 3504949 